\title{
INDUCTIVE INFERENCE FROM ALL POSITIVE AND SOME NEGATIVE DATA
}

$\operatorname{AUTHOR}(\mathrm{S})$ :

MOTOKI, Tatsuya

\section{CITATION:}

MOTOKI, Tatsuya. INDUCTIVE INFERENCE FROM ALL POSITIVE AND SOME NEGATIVE DATA. 数理解析研究所講究録 1991, 754: 35-44

ISSUE DATE:

1991-06

URL:

http://hdl.handle.net/2433/82119

RIGHT: 


\title{
INDUCTIVE INFERENCE \\ FROM ALL POSITIVE AND SOME NEGATIVE DATA
}

\author{
元木達也 (Tatsuya MOTOKI) \\ College of General Education, \\ Niigata University, \\ Ikarashi 2-8050, Niigata 950-21, Japan
}

\begin{abstract}
Keywords : Formal languages, computational learning, identification in the limit, usefulness of negative data
\end{abstract}

\section{Introduction}

Inductive inference is a fundamental component of intelligent behavior; so many researchers have discussed the computational aspect of induction process (see [2] for a survey). Gold [3] established a basic paradigm of inductive inference. He considers an induction process to be an infinite one that occasionally receives examples of some unknown rule, and simultaneously generates a sequence of guesses of the underlying rule; he then defines the process to make correct inference if the sequence of guesses eventually converges to a description of the underlying rule.

We are now concerned with inductive inference of (formal) languages, that is, those induction processes that (i) restrict the range of unknown rules to some fixed class of languages, ( $i i$ ) receive positive or negative facts $($, i.e., examples of elements in the unknown language, or examples of elements that are not in the language), and (iii) choose a guess language from the fixed class. Angluin [1] characterizes those classes of nonempty recursive languages that can be correctly infered when all and 
only positive facts are allowed to be eventually given to induction process. Shinohara [4, section 5.3] shows that when all positive facts are eventually given to induction process, finitely many more negative facts do not necessarily extend the possibility of correct inference.

In this paper, we consider negative facts to be the timely advice that observers give to induction process, and show that when all positive facts are eventually given to induction process, some supplementary negative facts might extend the possibility of correct inference. Hence, as we intuitionally accept it, we might construct an induction process of practical use by supposing the existence of teachers who help the process to make correct inference.

\section{Preliminaries}

In this section, we give basic definitions and a result about inductive inference of formal languages. Section 2.1 deals with the case where all and only positive facts are eventually given to induction process; section 2.2 deals with the case where all positive and some negative facts are eventually given.

\subsection{Inductive inference from positive data}

Let $\Sigma$ be a fixed nonempty finite set of symbols. We use $\Sigma *$ to denote the set of all finite strings of symbols from $\Sigma$, including the empty string $\varepsilon$, and $\Sigma^{+}$to denote the set $\Sigma^{*}-$ $\{\varepsilon\}$. A language is any subset of $\Sigma^{*}$. An indexed family of nonempty recursive languages is an infinite sequence $L_{1}, L_{2}, \ldots$ of nonempty languages such that the following membership function $f$ is computable:

$$
\begin{aligned}
f(i, w) & =1 & & \text { if } w \in L_{i} ; \\
& =0 & & \text { otherwise. }
\end{aligned}
$$


An inference machine $M$ is an effective procedure that occasionally requests an element in $\Sigma * x\{0,1\}$ as an input and occasionally outputs a positive integer as a guess. We regard an input $(s, 1)$ as a positive fact that $s$ is in the unknown language, and an input $(s, 0)$ as a negative fact that $s$ is not in the unknown language. To run the machine $M$ we provide with an infinite or finite sequence $\sigma=\left\langle\left(s_{1}, d_{1}\right),\left(s_{2}, d_{2}\right), \ldots\right\rangle$ of inputs; when $M$ requests an input for the $i$ th time, we supply with the ith element $\left(s_{i}, d_{i}\right)$. For each input sequence $\sigma$, we use $M[\sigma]$ to denote the infinite or finite sequence of guesses produced by $M$ on $\sigma$; for each finite sequence $\sigma$, we use $M(\sigma)$ to denote the last guess in $M[\sigma]$, that is, the most recent guess produced by $M$ on $\sigma$. Given an infinite sequence $\sigma$, we say $M[\sigma]$ converges to a positive integer $i$ if either $M[\sigma]$ is finite and the last guess is equal to $i$, or $M[\sigma]$ is infinite and all but finitely many terms in $M[\sigma]$ are equal to $i$.

A positive presentation of a nonempty language $L$ is an infinite sequence $\left\langle\left(s_{1}, 1\right),\left(s_{2}, 1\right), \ldots\right\rangle$ such that $\left\{s_{1}, s_{2}, \ldots\right\}$ $=L$. An inference machine $M$ is said to infer an indexed family of nonempty recursive languages $L_{1}, L_{2}, \ldots$ from positive data if for every $i \geq 1$ and every positive presentation $\sigma$ of $L_{i}$, the sequence $M[\sigma]$ converges to some positive integer $j$ such that $L_{j}=$ $L_{i}$. An indexed family of nonempty recursive languages $L_{1}, L_{2}, \ldots$ is said to be inferable from positive data if there exists an inference machine that infers the family $L_{1}, L_{2}, \ldots$ from positive data.

The following theorem characterizes when an indexed family of nonempty recursive languages is inferable from positive data.

Theorem 2.1 [1]. An indexed family of nonempty recursive languages $L_{1}, L_{2}, \ldots$ is inferable from positive data if and only if there exists an effective procedure that, given an input $i \geq 1$, enumerates a finite subset $T_{i}$ of $L_{i}$ such that for every $j \geq 1$ the condition $T_{i} \subseteq L_{j} \subseteq L_{i}$ implies $L_{j}=L_{i}$. 
2.2. Inductive inference from all positive and some negative data

Let $L_{1}, L_{2}, \ldots$ be an indexed family of nonempty recursive languages. A (negative) advisor is a function $A:\{1,2,3, \ldots\}$ $\longrightarrow 2^{\Sigma *}$ such that $A(i) \subseteq \bar{L}_{i}$ for every $i$. For each $i \geq 1$, we regard $A(i) \times\{0\}$ as a set of supplementary negative facts that observers will eventually give to the inference machine when the unknown language is $L_{i}$.

For each advisor $A$ and each $i \geq 1$, an $A$-positive presentation of $L_{i}$ is an infinite sequence $\left\langle\left(s_{1}, d_{1}\right),\left(s_{2}, d_{2}\right), \ldots\right\rangle$ of ordered pairs from $\Sigma * \times\{0,1\}$ such that $\left\{s \mid(s, 1)=\left(s_{k}, d_{k}\right)\right.$ for some $k\}=L_{i}$ and $A(i) \subseteq\left\{s \mid(s, 0)=\left(s_{k^{\prime}} d_{k}\right)\right.$ for some $\left.k\right\} \subseteq \bar{L}_{i}$. An inference machine $M$ is said to infer an indexed family of nonempty recursive languages $L_{1}, L_{2}, \ldots$ from positive data and an advisor $A$ if for every $i \geq 1$ and every $A$-positive presentation $\sigma$ of $L_{i}$, the sequence $M[\sigma]$ converges to some positive integer $j$ such that $L_{j}=L_{i}$. An indexed family of nonempty recursive languages $L_{1}, L_{2}, \ldots$ is said to be inferable from positive data and an advisor $A$ if there exists an inference machine that infers the family $L_{1}, L_{2}, \ldots$ from positive data and an advisor $A$.

\section{Usefulness of negative data}

We now establish a result that is similar to Theorem 2.1 .

Theorem 3.1. An indexed family of nonempty recursive languages $L_{1}, L_{2}, \ldots$ is inferable from positive data and an advisor $A$ if and only if there exists an effective procedure that, given an input $i \geq 1$, enumerates a finite subset $T_{i}$ of $L_{i}$ such that for every $j \geq 1$ the conjunction of $T_{i} \subseteq L_{j} \subseteq L_{i}$ and $A(j) \subseteq \bar{L}_{i}$ implies $L_{j}=L_{i}$

Proof. The proof proceeds in the similar manner as that of Theorem 2.1.

$(\Leftarrow)$ Suppose that there exists an enumeration procedure of 
$T_{i}$ as specified in the theorem, and consider the inference machine $M$ described as follows:

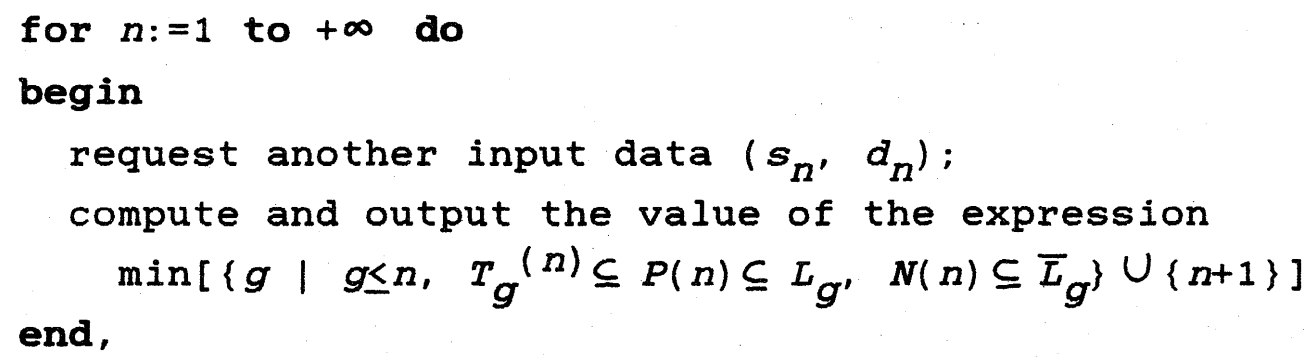

where we define $P(n)=\left\{s \mid(s, 1)=\left(s_{k}, d_{k}\right)\right.$ for some $\left.k \leq n\right\}$, define $N(n)=\left\{s \mid(s, 0)=\left(s_{k}, d_{k}\right)\right.$ for some $\left.k \leq n\right\}$, and use $T_{g}(n)$ to denote the set of strings produced in the first $n$ steps of the enumeration of $T_{g}$. [Note that the effectiveness of $M$ follows from the recursiveness of $L_{i}$ and the existence of enumeration procedure of $\left.T_{i} \cdot\right]$

To see that $M$ infers $L_{1}, L_{2}, \ldots$ from positive data and an advisor $A$, let $j \geq 1$ be arbitrary and assume that $\sigma=<\left(s_{1}, d_{1}\right)$, $\left(s_{2}, d_{2}\right), \ldots>$ is any A-positive presentation of $L_{j}$. It remains to show that $M[\sigma]$ converges to some $i$ such that $L_{j}=L_{i}$. For each $n \geq 1$, we use $\sigma(n)$ to denote the initial subsequence $<\left(s_{1}\right.$, $\left.d_{1}\right), \ldots,\left(s_{n}, d_{n}\right)>$.

Since $T_{j}$ is a finite subset of $L_{j}$, we have $T_{j} \subseteq P(n)$ for sufficiently large $n$; so define $n_{0}=\min \left\{n \mid T_{j} \subseteq P(n)\right\}$. Then we can easily prove two claims: (i) $M(\sigma(n)) \leq j$ for every $n \geq n_{0}$, and (ii) $M(\sigma(n)) \leq M(\sigma(m))$ for every $m \geq n \geq n_{0}$. From these claims we see that $M[\sigma]$ converges to some positive integer, say $i$. Thus there must exists an integer $n^{*}$ such that for every $n \geq n^{*}$,

$$
\begin{aligned}
& T_{i}(n) \subseteq P(n) \subseteq L_{i} \quad \text { and } \\
& N(n) \subseteq \bar{L}_{i} ;
\end{aligned}
$$

so by letting $n \longrightarrow \infty$ two relations

$$
\begin{aligned}
& T_{i} \subseteq \bigcup_{n=1}^{\infty} P(n) \subseteq L_{i} \quad \text { and } \\
& U_{n=1}^{\infty} N(n) \subseteq \bar{L}_{i}
\end{aligned}
$$


follow. Since we obtain $\bigcup_{n=1}^{\infty} P(n)=L_{j}$ and $A(j) \subseteq \bigcup_{n=1}^{\infty} N(n)$ from the choice of $\sigma$ and the definitions of $P(n)$ and $N(n)$, we now derive from (1), (2) two relations $T_{i} \subseteq L_{j} \subseteq L_{i}$ and $A(j) \subseteq \bar{L}_{i}$; so from the choice of $T_{i}$ we deduce the required equation $L_{j}=L_{i}$.

$(\Longrightarrow)$ Suppose $M$ is an inference machine that infers $L_{1}$, $L_{2}, \ldots$ from positive data and an advisor $A$. For each $i \geq 1$, let $\left(s_{i, 1}, d_{i, 1}\right),\left(s_{i, 2}, d_{i, 2}\right), \ldots$ be an enumeration of $L_{i} \times\{1\} \cup \bar{L}_{i} \times\{0\}$, and let $P_{i, 1}, \rho_{i, 2}, \ldots$ be an enumeration of all finite sequences of elements from $L_{i} \times\{1\} \cup \bar{L}_{j} \times\{0\}$. [Note that effective enumeration procedures for $\left(s_{i, m^{\prime}} d_{i, m}\right)^{\prime} s$ and $P_{i, m}{ }^{\prime} s$ are seen to exist from the existence of effective enumeration procedure of $\Sigma *$ and the recursiveness of $L_{i} \cdot$ ]

To enumerate for any given $i \geq 1$ the required finite subset $T_{i}$ of $L_{i}$, consider the infinite procedure $P(i)$ described as follows:

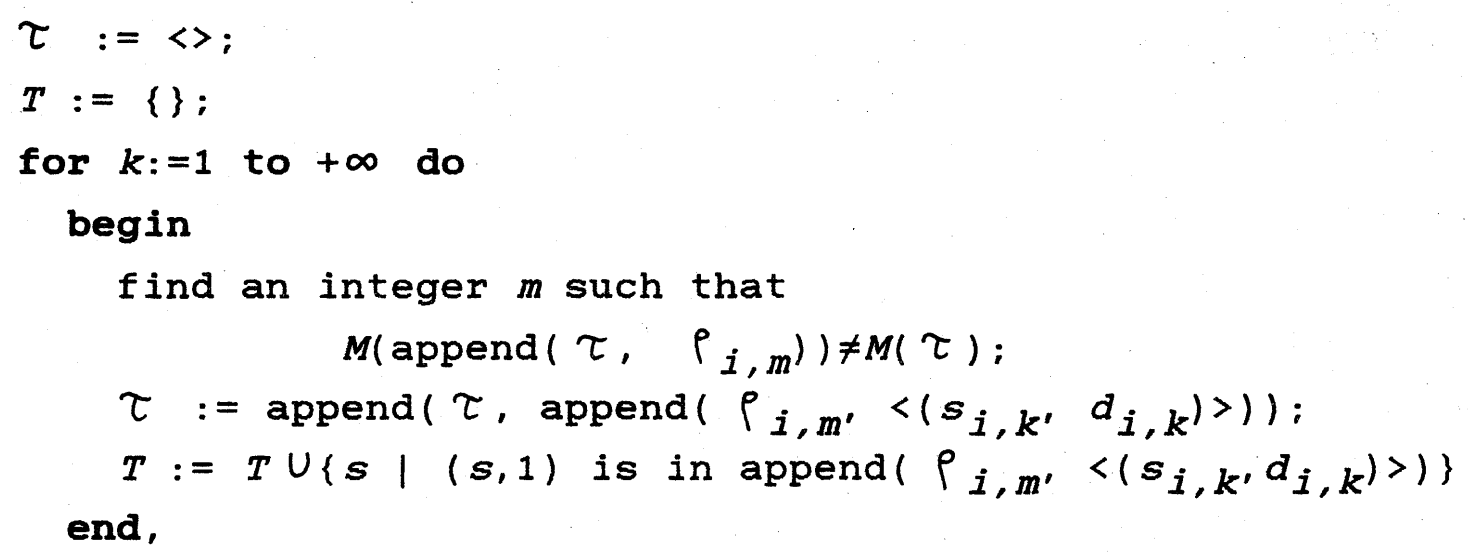

where we use $\ll$ to denote the null sequence, and append $\tau, \rho$ ) to denote the sequence obtained by appending sequence $\rho$ to the end of finite sequence $\tau$. Now let $T_{i}$ be the limiting value of $T$ in $P(i)$; similarly let $\tau(i)$ be the limiting value of $\tau$ in $P(i)$. We easily see $T_{i} \subseteq L_{i}$, and can regard $P(i)$ as an effective procedure to enumerate $T_{i}$.

To show the finiteness of $T_{i}$, let us assume the opposite and see what happen. Since each $\rho_{i, m}$ is finite, the execution of $P(i)$ goes infinitely many times around the for loop; so $\tau(i)$ is 
an A-positive presentation of $L_{i}$. However, we can also derive the consequence that the execution of $M$ on $\tau(i)$ changes its guess infinitely many times and so $M[\tau(i)]$ does not converge to any integer. Therefore $M$ fails to make correct inference when we provide with an input sequence $\tau(i)$, a contradiction.

The argument in the preceding paragraph also shows that the execution of $P(i)$ cannot go infinitely many times around the for loop. Hence after the execution of $P(i)$ goes some finite times around the for loop, the procedure $P(i)$ cannot find an integer $m$ such that $M$ (append $\left.\left.\tau, P_{i, m}\right)\right) \neq M(\tau)$; after that time, program variables $\tau$ and $T$ have already converged to $\tau(i)$ and $T_{i}$ respectively. Therefore $\tau(i)$ satisfies the condition

$$
M\left(\text { append }\left(\tau(i), \quad \rho_{i, m}\right)\right)=M(\tau(i)) \text { for every } m \geq 1
$$

Finally, let $j \geq 1$ be arbitrary and assume that two conditions

$$
\begin{aligned}
& T_{i} \subseteq L_{j} \subseteq L_{i} \quad \text { and } \\
& A(j) \subseteq \bar{L}_{i}
\end{aligned}
$$

hold. Now it remains to deduce the equation $L_{i}=I_{j}$. To do this, consider two input sequences

$$
\begin{aligned}
& \sigma_{1} \text { =append }\left(\tau(i),\left\langle\left(s_{i, 1}, d_{i, 1}\right),\left(s_{i, 2}, d_{i, 2}\right), \ldots\right\rangle\right) \text { and } \\
& \sigma_{2} \text { =append }\left(\tau(i),\left\langle\left(t_{1}, e_{1}\right),\left(t_{2}, e_{2}\right), \ldots>\right),\right.
\end{aligned}
$$

where $\left(t_{1}, e_{1}\right),\left(t_{2}, e_{2}\right), \ldots$ denote an enumeration of $L_{j} \times\{1\}$ $\cup \bar{L}_{i} \times\{0\}$. Obviously $\sigma_{1}$ is an A-positive presentation of $L_{i}$; from (4), (5) and the equation $\{s \mid(s, 1)$ is in $\tau(i)\}=T_{i}$, the sequence $\sigma_{2}$ is seen to be an $A$-positive presentation of $L_{j}$. So $M\left[\sigma_{1}\right]$ converges to some integer $i^{*}$ such that $L_{i *}=L_{i}$; similarly $M\left[\sigma_{2}\right]$ converges to some integer $j^{*}$ such that $L_{j^{*}}=L_{j}$.

Now, observe that for each $p \geq 1$ two equations 


$$
\begin{array}{r}
M\left(\text { append }\left(\tau(i),<\left(s_{i, 1}, d_{i, 1}\right), \ldots,\left(s_{i, p}, d_{i, p}\right)>\right)\right) \\
=M(\tau(i)) \text { and } \\
M\left(\text { append }\left(\tau(i),<\left(t_{1}, e_{1}\right), \ldots,\left(t_{p}, e_{p}\right)>\right)\right)=M(\tau(i))
\end{array}
$$

follows from (3). From these equations, we deduce that both $M\left[\sigma_{1}\right]$ and $M\left[\sigma_{2}\right]$ must converge to the integer $M(\tau(i))$, that is, $i^{*}=j^{*}=M(\tau(i))$; and hence we obtain the required equation $L_{i}=L_{i *}$ $=L_{j *}=L_{j}$

We next give an example of the application of Theorem 3.1.

Example 3.2. Let $\Sigma=\{a, b\}$, and let $h$ be a computable bijection from $\{1,2,3, \ldots\}^{2}$ to $\{1,2,3, \ldots\}$. Now consider the family $L_{1}, L_{2}, \ldots$ and an advisor $A$ defined as follows:

$$
\begin{aligned}
& L_{h(i, j)}=\{a\}^{+} U\{b\}^{+} \quad \text { if } i=j=1 ; \\
& =\{a\}^{+} \cup\left\{b, b^{2}, \ldots, b^{j-1}\right\} \quad \text { if } i=1, j>1 \text {; } \\
& =\left\{a, a^{2}, \ldots, a^{i-1}\right\} \cup\{b\}^{+} \quad \text { if } i>1, j=1 \text {; } \\
& =\left\{a, a^{2}, \ldots, a^{i-1}\right\} \cup\left\{b, b^{2}, \ldots, b^{j-1}\right\} \text { if } j>1, j>1 \text {; } \\
& A(h(i, j))=\{\} \quad \text { if } i=j=1 \text {; } \\
& =\left\{b^{j}\right\} \quad \text { if either } 1<i \leq j \text {, or } 1=i<j \text {; } \\
& =\left\{a^{i}\right\} \text { if either } 1<j<i \text {, or } 1=j<i \text {; }
\end{aligned}
$$

Then by setting the "telltale set"

$$
\begin{aligned}
T_{h(m, n)} & =\{\} & & \text { if } m=n=1 ; \\
& =\left\{a^{n}\right\} & & \text { if } m=1, n>1 ; \\
& =\left\{b^{m-1}\right\} & & \text { if } m>1, n=1 ; \\
& =L_{h(m, n)} & & \text { if } m>1, n>1 ;
\end{aligned}
$$

and applying Theorem 3.1, we derive the consequence that $L_{1}$, $L_{2}, \ldots$ is inferable from positive data and an advisor $A$. [Therefore the class $L_{1}, L_{2}, \ldots$ is considered to be inferable from positive data and one supplementary negative data.] In fact, let 


$$
\begin{aligned}
& C(h(m, n))=\left\{L_{h(i, j)} \mid T_{h(m, n)} \subseteq L_{h(i, j)} \subsetneq L_{h(m, n)},\right. \\
& \left.A(h(i, j)) \subseteq \bar{L}_{h(m, n)}\right\} \text {. }
\end{aligned}
$$

Then for each $m, n>1$ we have

$$
\begin{aligned}
& C(h(1,1)) \\
& =\left\{L_{h(i, j)} \mid(i, j) \neq(1,1), A(h(i, j)) \subseteq \overline{\{a\}^{+} \cup\{b\}^{+}}\right\} \\
& =\{\} \\
& C(h(m, 1)) \\
& =\left\{L_{h(i, j)} \mid b^{m-1} \in L_{h(i, j)} \varsubsetneqq L_{h(m, 1)}, A(h(i, j)) \subseteq \bar{L}_{h(m, 1)}\right\} \\
& =\left\{L_{h(i, j)} \mid(m \leq j \text { or } j=1), 1<i \leq m,(i, j) \neq(m, 1)\right. \text {, } \\
& =\left\{L_{h(i, j)} \mid \text { either }\left(1<i \leq m \leq j, b^{j} \in \bar{L}_{h(m, 1)}\right)\right. \\
& \left.A(h(i, j)) \subseteq \bar{L}_{h(m, 1)}\right\} \\
& \text { or } \left.\left(j=1,1<i<m, a^{i} \in \bar{L}_{h(m, 1)}\right)\right\} \\
& =\{\} \\
& C(h(1, n)) \\
& =\left\{L_{h(i, j)} \mid a^{n} \in L_{h(i, j)} \subsetneq L_{h(1, n)}, A(h(i, j)) \subseteq \bar{L}_{h(1, n)}\right\} \\
& =\left(L_{h(i, j)} \mid(n<i \text { or } i=1), 1<j \leq n,(i, j) \neq(1, n)\right. \text {, } \\
& =\left\{L_{h(i, j)} \mid \text { either }\left(1<j \leq n<i, a^{i} \in \bar{L}_{h(1, n)}\right)\right. \\
& \left.A(h(i, j)) \subseteq \bar{L}_{h(1, n)}\right\} \\
& =\{\} \\
& \text { or }\left(i=1,1<j<n, b^{\left.\left.j \in \bar{L}_{h(1, n)}\right)\right\}}\right. \\
& C(h(m, n)) \\
& =\left\{L_{h(i, j)} \mid L_{h(m, n)} \subseteq L_{h(i, j)} \subsetneq L_{h(m, n)}\right. \text {. } \\
& =\{\} \\
& \left.A(h(i, j)) \subseteq \bar{L}_{h(m, n)}\right\}
\end{aligned}
$$

From Theorem 3.1 and Example 3.2, we have the conclusion that when all positive data are eventually given to inference machine, some negative data might extend the possibility of correct inference; by receiving some negative advice from teachers, machine can develop its ability to make correct inference. Thus we might construct an inference machine of 
practical use by supposing the existence of teachers who help the machine to make correct inference.

\section{Acknowledgment}

The author would like to thank Dr. T. Shinohara for his valuavle comments, and Prof. T. Nishizawa for drawing author's attention to this research area.

\section{References}

[1] D. Angluin, Inductive inference of formal languages from positive data, Inform. and Control 45 (1980) 117-135.

[2] D. Angluin and C. H. Smith, Inductive inference: theory and methods, Comput. Survey 15 (1983) 237-269.

[3] E. M. Gold, Language identification in the limit, Inform. and Control 10 (1967) 447-474.

[4] T. Shinohara, studies on inductive inference from positive data, doctoral thesis, Kyushu University, Japan (1986). 